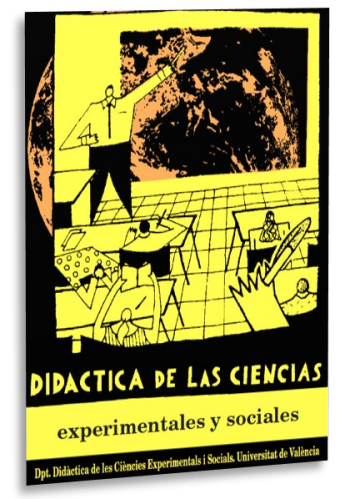

\title{
Experimentando con los sentidos: un rincón de ciencias en Educación Infantil
}

\author{
Experimenting with the senses: a science \\ corner in Pre-School Education
}

DOI: 10.7203/DCES.39.16893

\author{
María Puig Gutiérrez \\ Universidad de Sevilla, mpuig@us.es \\ ORCID iD: https://orcid.org/0000-0002-7536-2976 \\ Lidia López-Lozano \\ Universidadde Huelva, lidia.lopez@ddi.uhu.es \\ ORCID iD: https://orcid.org/0000-0002-5181-638X \\ Rocío García Rodríguez \\ Universidad de Sevilla, rocio.garo@hotmail.com
}

\begin{abstract}
RESUMen: Desde la Didáctica de las Ciencias se señala la idoneidad y necesidad de trabajar ciencias desde las edades más tempranas. Bajo esa premisa, se plantea una propuesta didáctica para trabajar la temática de los sentidos basada en el constructivismo que adopta la metodología de rincones. Se detallan las 5 actividades de carácter libre que conforman este rincón, una por cada sentido. La intervención se desarrolló en un centro de Educación Infantil de la provincia de Sevilla. La experiencia ha resultado atractiva para el alumnado por la multitud y diversidad de materiales usados y por las cuestiones planteadas. La dinámica de las actividades les ha dotado de mayor autonomía en su proceso de experimentación, asimismo se aprecia el paso de su desarrollo inicial, esencialmente individual, a uno colectivo. Concluimos que el carácter abierto de las actividades y las preguntas que las desencadenan resultan claves en los procesos de indagación experimentados.
\end{abstract}

Palabras Clave: Educación Infantil, educación científica, actividades escolares, investigación educativa, experiencia pedagógica.

ABSTRACT: The Didactic of Sciences points out the suitability and need to explore sciences from an early age. Under this premise, we describe a didactic proposal to work on the topic of "the senses" based on constructivism that adopts the methodology of corners. The five activities, one for each sense, that make up this corner are detailed. The intervention was carried out in a Pre-School Education center in the province of Seville. The experience was attractive to the students due to the multitude and diversity of materials used and inquiries raised. The dynamics of these activities gave them greater autonomy in their experimentation process. It is possible to see a growth in their development that changes from an individual one to a collective one. We conclude that the open nature of the activities and the questions that trigger them have been key in promoting the processes of inquiry undergone.

KEYWORDS: Early Childhood Education, science education, school activities, educational research, teaching experience.

Fecha de recepción: marzo de 2020 Fecha de aceptación: junio de 2020

Agradecemos desde estas líneas la disponibilidad y colaboración prestada por la Escuela de Educación Infantil "El Real" ubicada en la localidad de Carmona (Sevilla), en la que se desarrolló la experiencia. 


\section{INTRODUCCIÓN}

Numerosos autores (Eshach y Fried, 2005; García-Carmona, Criado y Cañal, 2014; GómezMontilla y Ruiz-Gallardo, 2016; Torres-Porras, Alcántara, Arrebola, Rubio y Mora, 2017; Saçkes, Trundle, Bell y O'Connell, 2011) advierten la necesidad e idoneidad de enseñar y aprender ciencias desde edades tempranas, de manera que desde la etapa de Infantil se propicien las primeras experiencias científicas de los más pequeños. Gómez-Montilla y Ruiz-Gallardo (2016) recogen varias razones que lo apoya. Algunas responden a las características de los niños: acciones como observar y pensar sobre la naturaleza les resultan divertidas y son capaces de comprender conceptos científicos. Además, se desarrollan actitudes positivas hacia la ciencia, su exposición facilita una mejor comprensión de los conceptos que abordarán posteriormente y la ciencia se presenta como una eficiente manera de desarrollar el pensamiento científico. Estos autores, también, lo relacionan con el mundo altamente tecnificado en el que están creciendo. Por tanto, se trata de ofrecer propuestas que se presten a dar explicaciones a lo que ocurre a su alrededor. Asimismo, por las propias características del aula de Infantil, tal y como apuntan Blanco-Carabias, López-Luengo, Vallés y Gil (2018, p. 1116) "el interés de los procedimientos científicos en esta etapa es dotar de un instrumento que capacite hacia la canalización de sensaciones y permita establecer así un conocimiento razonado que se estructura mediante su propio lenguaje infantil".

Concretando sobre esto último, en la Educación Infantil, es fundamental el desarrollo sensorial, puesto que la información que llega a través de los sentidos es esencial para el desarrollo de las funciones mentales en el niño, dependiendo así la actividad cerebral de estímulos sensoriales. Por ello, decimos que la base del aprendizaje en los primeros años es perceptual (Mateo, Ferrer, Mazas, Hervás y Muñoz, 2017). El niño manifiesta una gran necesidad de explorar lo que le rodea a través de los sentidos, tocar, morder, escuchar, olfatear, es la manera de acercarse a lo desconocido y aprender de ello. De este modo, cobra protagonismo la educación sensorial que implica no sólo conocer los órganos de los sentidos, sino experimentar con ellos comprobando que nos permiten conocernos a nosotros mismos y al mundo que nos rodea (Aranda, 2008). Según Johansson y Løkken (2014) la forma en que vivimos, comprendemos y nos comunicamos con nuestros sentidos siempre implican relaciones sociales. Cuando experimentamos, vemos, oímos, olfateamos o tocamos otras cosas, estamos siempre rehaciéndonos a nosotros mismos. Es por ello por lo que las experiencias sensoriales, así como su posterior reflexión proporcionan nuevos niveles de conciencia, convirtiendo al cuerpo humano en el centro de todas nuestras sensaciones.

Proporcionar experiencias diversas que ejerciten la percepción a través de cada uno de los sentidos, ayudará al niño a discriminar y distinguir las propiedades de los objetos, ayudándoles a intervenir sobre ellos (Córdoba, 2011; Moren, 2015). Por lo tanto, de acuerdo con Mateo et al. (2017), los maestros deben plantear experiencias que les permita explorar su entorno a través de los sentidos, puesto que "no basta con ver, oler, oír, saborear y tocar, es necesario aprender a hacerlo a la manera humana, es decir, de manera consciente y comprometida con el mundo en el que vivimos" (p. 952).

Desde estas consideraciones, apostamos por crear espacios de aprendizaje de las ciencias donde el alumnado pueda experimentar por sí mismo y en interacción con los demás (Cruz-Guzmán, GarcíaCarmona y Criado, 2017; García-Carmona et al., 2014).

Generalmente, a esta idea se suele unir el juego como principal motor de aprendizaje en esta etapa educativa (Bergen, 2002; Bermejo y Blázquez, 2016; Rogers, 2011; Singer, Golinkoff y HirshPasek, 2006). Así, de acuerdo con Fernández-Oliveras, Molina y Oliveras (2016), lo que se aprende de forma lúdica permanece en el tiempo, más aún si se aprende a través de la manipulación y la experimentación, en lugar de completando una ficha.

En este sentido, consideramos que los rincones son una buena forma de aunar las citadas características. Los rincones son zonas del aula delimitadas, en las que se trabajan tareas de forma simultánea, de manera individual o grupal (Madrid y Mayorga, 2012). Podemos decir, por tanto, de acuerdo con Rodríguez-Marín y Puig (2018, p. 24) que "los rincones son espacios organizados y 
planificados con una intencionalidad educativa, dotados de recursos que ayudan al alumnado a alcanzar los objetivos propuestos" ofreciendo la posibilidad de que el alumno aprenda de manera autónoma. Además, esta metodología permite responder a los intereses, diferencias y ritmos de cada uno de los niños mediante estrategias organizativas (Martín, 2008). Según Laguía y Vidal (2011) se trata de una estrategia didáctica que intenta fomentar que el niño vaya construyendo su conocimiento mientras que participa de forma activa.

Encontramos diversas experiencias que manifiestan las ventajas de esta metodología aplicada especialmente a la enseñanza de las ciencias. Así podemos destacar los beneficios del denominado rincón de ciencias o de los experimentos como un espacio idóneo desde el que acercar a los más pequeños al mundo científico a través de la experimentación (Cabello 2011; Cruz-Guzmán, 2018; Fernández-Arroyo, López-Lozano y García-Díaz, 2018; Fernández Manzanal y Rodríguez-Barreiro, 2006; Gómez-Crespo, 2003; Gómez-Montilla y Ruiz-Gallardo 2016; Medrano, Bello y Fernández, 2006; Ramos y Pérez, 2009).

Al proponer trabajar por rincones debemos hacer referencia a las denominadas actividades libres o centradas en el niño. Se trata de actividades que no requieren de la intervención del adulto durante su desarrollo y que, de acuerdo con Epstein (2014), cumplen con las siguientes características:

- Favorecen que los niños investiguen cómo funcionan las cosas mediante la exploración activa de materiales, acciones e ideas.

- Potencian el establecimiento de relaciones entre los propios niños y facilitan la ayuda mutua.

- Motivan al alumnado a resolver problemas por sí mismos y hacen posible el desarrollo de nuevas habilidades partiendo de los desafíos y retos que van surgiendo entre ellos mismos a lo largo del proceso.

Investigaciones recientes indican que este tipo de actividades favorecen la flexibilidad cognitiva (Ansari y Purtell, 2017), el interés del niño (Andiema, 2016) y la imaginación (Diachenko, 2011). Concretamente en el aprendizaje científico, numerosas investigaciones ensalzan la necesidad de iniciar a los más pequeños en las ciencias a través de la experimentación, permitiéndoles formular sus propias hipótesis, indagar y hallar respuestas a las cuestiones planteadas (Cruz-Guzmán, GarcíaCarmona y Criado, 2017; De la Blanca, Hidalgo y Burgos, 2013; Zacharia, Loizou y Papaevripidou, 2012). En definitiva, estamos ante una forma de trabajo clave para acercar al alumnado de Infantil a la ciencia.

\section{OBJETIVOS}

Bajo estos supuestos, nos planteamos diseñar y valorar la puesta en práctica de una secuencia didáctica cuyo fin es fomentar y estimular el desarrollo sensorial y las habilidades de indagación (observación, cuestionamiento, formulación de preguntas y predicciones, experimentación e interacción con su entorno e imaginar posibles soluciones) del alumnado de Infantil a través de rincones de trabajo.

Los objetivos que persigue la propuesta didáctica son los siguientes:

- Desarrollar el sentido del tacto, la vista, el oído, el gusto y el olfato. En consecuencia, discriminar y asociar sabores, olores, sonidos, colores y texturas.

- Desarrollar las habilidades de indagación y experimentación. Por tanto, formular que estas preguntas sirvieron para introducir la acción de vibrar, el concepto de vibración y comprobar resultados.

- Desarrollar la coordinación óculo-manual.

Además de potenciar la imaginación y creatividad, el trabajo en equipo y fomentar la autonomía. 


\section{Metodología}

\subsection{Participantes y contexto}

La propuesta didáctica que se plantea se lleva a cabo en un Centro de Educación Infantil de la provincia de Sevilla, en un aula formada por 26 alumnos de 4 y 5 años. El alumnado estaba habituado a trabajar según un modelo tradicional, donde el docente indicaba lo que tenían que hacer e iba dando las pautas de actuación, por lo que, no solían tener posibilidad de elegir el tipo de actividad que querían hacer, ni realizar experiencias de indagación y experimentación libremente. Los diferentes rincones creados se fueron sucediendo a lo largo de ocho semanas, de manera que se dedica aproximadamente una semana y media a cada uno de los sentidos. El desarrollo de estos rincones sensoriales se produjo de manera simultánea a otros rincones de juego existentes en el aula, quedando a elección de los niños los rincones que visitar. Para garantizar que todo el alumnado a lo largo de la semana y media visite todos los rincones disponibles en el aula, se utiliza una tabla de registro de doble entrada en la que aparecen los nombres de los rincones representados con diferentes iconos y los nombres de los niños. El alumnado iba completando los rincones, estando en cada uno grupos de entre 5 y 6 niños.

\subsection{Descripción de la propuesta}

La propuesta didáctica se apoya en el constructivismo y en la indagación adoptando la metodología de rincones de actividad. Concretamente, se centra en el desarrollo de actividades de carácter libre. Los contenidos abordados están recogidos en el mapa que se muestra en la Figura 1.

FIGURA 1. Mapa de contenidos sobre los sentidos

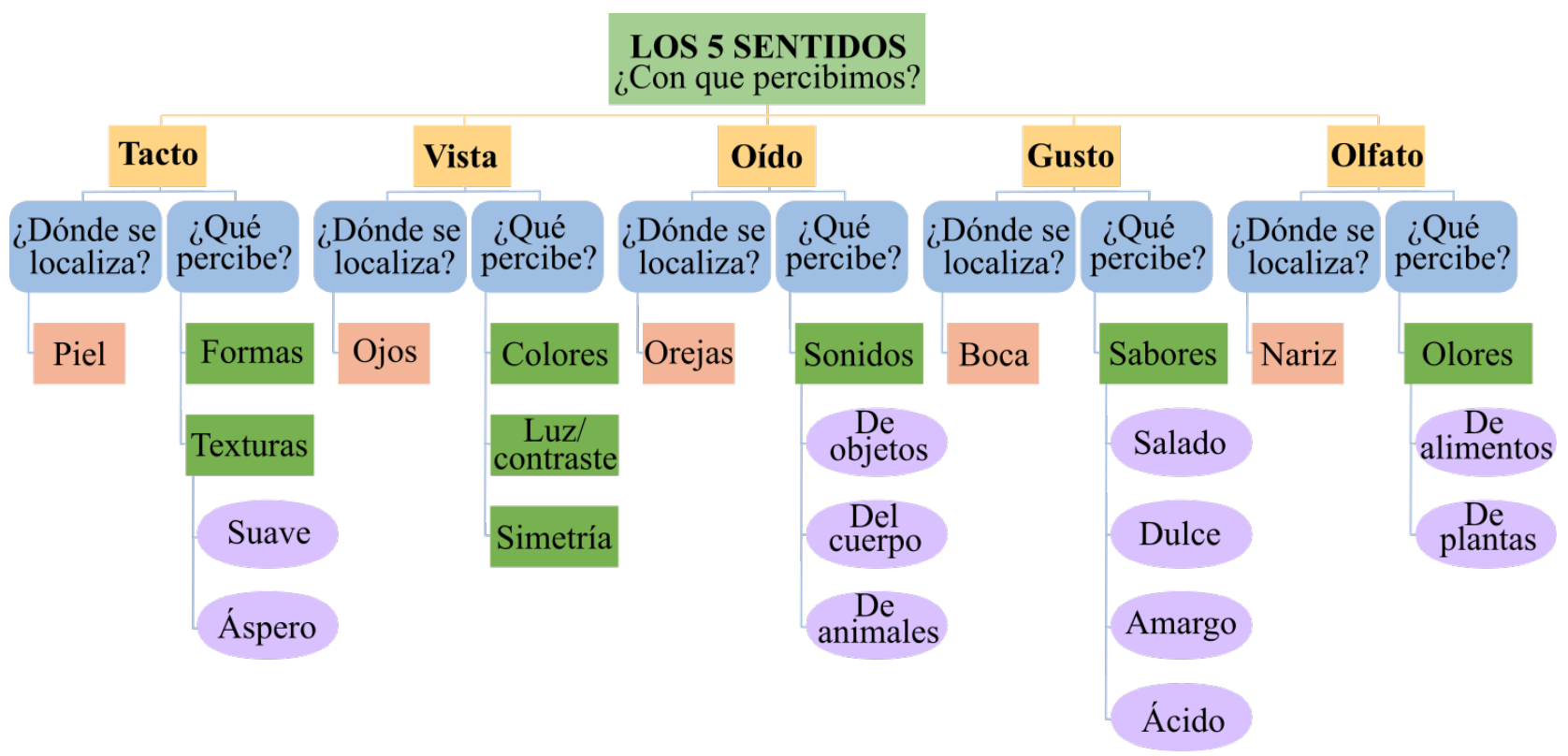

Fuente: Elaboración propia

En las Tablas 1-5 mostramos algunas de las actividades realizadas para cada uno de los cincos rincones, uno por sentido, que integran la propuesta. Se describen cada una de ellas y se muestra la pregunta que desencadena la actividad junto a un listado de los materiales necesarios. Durante el desarrollo de la experiencia se llevaron a cabo más actividades de las aquí recogidas, pero por limitación de espacio, hemos seleccionado las más significativas. 
TABLA 1. Actividades realizadas en el rincón del tacto

\begin{tabular}{|c|c|}
\hline Pregunta y descripción & Materiales \\
\hline $\begin{array}{l}\text { ¿Cómo puedo volver blando un trozo de barro duro? } \\
\text { Se ofrecen algunos trozos de barro duro y se les pregunta a los } \\
\text { niños y niñas si pueden hacer que se ponga más blando. Se } \\
\text { ofrecen diferentes materiales con los que pueden probar. }\end{array}$ & $\begin{array}{l}\text { 1.Trozos de barro duro; 2. Agua; } \\
\text { 3. Vaselina; 4. Aceite; } 5 . \\
\text { Secador; } 6 . \text { Mazo. }\end{array}$ \\
\hline $\begin{array}{l}\text { ¿Qué hay en la caja de las sorpresas? } \\
\text { Los niños introducen las manos en la caja y, a través del tacto, } \\
\text { adivinan cuáles de los objetos que aparecen en las tarjetas están } \\
\text { dentro de la caja. }\end{array}$ & $\begin{array}{l}\text { 1.Caja de la sorpresa elaborada } \\
\text { con madera y una bolsa de tela; } 2 \text {. } \\
\text { Objetos de la vida cotidiana } \\
\text { (esponja, cepillo, gafas de sol, } \\
\text { lápiz, etc.) }\end{array}$ \\
\hline $\begin{array}{l}\text { ¿Qué hay dentro de los globos? } \\
\text { Se proporciona a los niños globos rellenos de diferentes } \\
\text { elementos. Al mismo tiempo se ofrece en diferentes recipientes } \\
\text { estos elementos. Los niños y niñas a través del tacto van } \\
\text { averiguando qué hay dentro de los globos. }\end{array}$ & 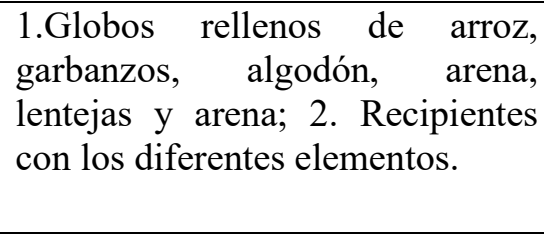 \\
\hline $\begin{array}{l}\text { ¿Cómo podemos clasificar estos materiales? } \\
\text { Se ofrecen diferentes materiales diversos y un par de bandejas } \\
\text { vacías, los niños y niñas van clasificando los materiales en } \\
\text { función de los criterios que decidan. }\end{array}$ & $\begin{array}{l}\text { 1.Diferentes materiales: algodón, } \\
\text { terciopelo, lija, lana, estropajo, } \\
\text { corteza de árbol, etc.; } 2 \text {. Dos } \\
\text { bandejas vacías. }\end{array}$ \\
\hline
\end{tabular}

Fuente: Elaboración propia

TABLA 2. Actividades realizadas en el rincón de la vista

Pregunta y descripción
¿Qué hay en la botella?
En una botella de plástico transparente se introducen diferentes
objetos como por ejemplo lápices, botones..., después se le
añade sal y se cierra. Los niños y niñas van averiguando al
moverla que objetos se encuentran escondidos en ella. Cada vez
que descubren un nuevo objeto buscan la tarjeta
correspondiente, a fin de trabajar la discriminación visual en la
búsqueda de objetos iguales.

¿Qué vemos con la lupa?

Se ofrece una bandeja con lupas y diferentes objetos cotidianos y de la naturaleza. Los niños y niñas exploran (observan y manipulan) estos objetos con la lupa, pudiendo buscar objetos nuevos para ver a través de ella.

¿Qué hay en las radiografías?

Con una mesa de luz casera y distintas radiografías, los niños y niñas pueden descubrir qué hay en una radiografía. La mesa de luz permite al mismo tiempo experimentar con otros objetos comprobando las diferencias con las radiografías.

¿Cómo podemos crear nuevos colores?

Con una rueda en la que se elaboran distintas ventanas de diversos colores de papel celofán, los niños y niñas van girando las ventanas y experimentando con los colores, comprobando si es posible conseguir colores nuevos a partir de los existentes. Pueden al mismo tiempo hacer comprobaciones con pintura de dedos.

\section{Materiales}

1.Botella de plástico transparente;

2. Objetos diversos pequeños: botones, pinzas de la ropa, gomas de borrar, sacapuntas, etc.; 3. Tarjetas con las imágenes de los objetos.

1.Lupas; 2. Objetos cotidianos y de la naturaleza: flores, piña, palos, bolígrafos, etc.

1.Mesa de luz; 2. Radiografías de partes del cuerpo humano.

1.Cartulinas; 2. Papel celofán de colores; 3. Pinturas de dedos. 
TABLA 3. Actividades realizadas en el rincón del oído

\begin{tabular}{ll}
\hline Pregunta y descripción & Materiales \\
\hline Iniciando la indagación ¿Todos los objetos producen sonidos? & 1. Botes opacos; 2. Objetos \\
Los niños y niñas buscan entre los objetos que les rodean para tratar de & $\begin{array}{l}\text { cotidianos presentes en el aula. } \\
\text { ver si todos pueden producir sonidos. }\end{array}$
\end{tabular}

¿Qué sonidos produce nuestro cuerpo?

1.Fonendoscopio.

Se ofrece a los niños y niñas un fonendoscopio y se indica que deben escuchar los diferentes sonidos de sus cuerpos, con o sin este instrumento.

¿Qué hay dentro?

Creamos con los niños diferentes botes opacos en los que introducimos distintos materiales. Posteriormente pueden tratar de averiguar qué material hay dentro de cada bote escuchando como suena al agitarlo.

¿Cómo suenan?

Ofrecemos a los niños diferentes cilindros de cartón y distintos tubos de tubería, así como otros objetos como globos, cinta adhesiva y tijeras. Les preguntamos si el sonido se transmite a través de estos materiales y si escuchamos igual un sonido a través de ellos que sin ellos. Los niños y niñas van haciendo sus propias comprobaciones, haciendo sonar los diferentes elementos y explorando el modo en el que se trasmite el sonido a través de ellos. Ponemos, además, al alcance de los niños y niñas algunos elementos que emiten sonidos para que comparen y hagan más comprobaciones: despertador, altavoz, etc.

1.Botes opacos; 2. Materiales diversos: arroz, pinzas de la ropa, harina, lápices algodón, etc.

1.Cilindros de cartón, tubos de tuberías; 2 . Tijeras, globos y cinta adhesiva; 3 . Despertador y altavoz.

Fuente: Elaboración propia

TABLA 4. Actividades realizadas en el rincón del gusto

Pregunta y descripción
¿A qué sabe?
Se presentan en diversos platos alimentos salados, dulces, ácidos y
amargos y varias bandejas vacías. Preguntamos a los niños y niñas si
todos los alimentos de los platos saben igual, y si algunos se parecen
entre ellos. Así les indicamos que sería conveniente organizar los
alimentos en función de su parecido en sabor.
¿De qué es este zumo?
Se ofrece a los niños y niñas diversos vasos opacos con distintos zumos
de fruta en su interior. Al mismo tiempo se muestran las frutas de las
que proceden los zumos. Los niños y niñas deben averiguar de qué
fruta está hecho cada uno de los zumos que hay en los vasos.

¿Cómo podemos cambiar el sabor del agua?

Materiales

1.Platos y bandejas; 2.

Alimentos variados: galletas

saladas, chocolate, golosinas, aceite, limón, etc.

Los niños y niñas disponen de distintos vasos con agua y a la vez se les ofrecen diferentes elementos como sal, azúcar, limón, naranja. Pueden ir probando que le ocurre al sabor del agua cuando le echan algunos de estas sustancias.

¿Cómo podemos hacer que algo cambie su aspecto, pero no su sabor? Se ofrece a los niños y niñas diversos yogures naturales y colorante alimentario de distintos tonos. Les preguntamos si los yogures tendrán el mismo sabor al mezclarlos con los diferentes colorantes. El objetivo es que discriminen el sabor sin dejarse influir por el color/aspecto. 1.Vasos opacos; 2. Zumos naturales distintos: piña, melocotón, naranja, manzana, etc.; 3. Fruta variada: piña, melocotón, naranja, manzana, etc.

1.Vasos con agua; 2. Sal; 3.

Azúcar; 4. Limón; 5. Naranja

Realizarán para ello las comprobaciones que consideren necesarias.

1.Yogures naturales; 2.
Colorante alimentario de
diferentes tonos; 3. Cuencos y
cucharas.

Fuente: Elaboración propia 
TABLA 5. Actividades realizadas en el rincón del olfato

\begin{tabular}{l}
\hline Pregunta y descripción \\
\hline ¿Cómo podemos hacer bolsitas de olores diferentes? \\
Se ofrece a los niños y niñas diferentes plantas aromáticas para que \\
eligiendo las que quieren elaboren sus propias bolsitas aromáticas. \\
\hline ¿A qué huele? \\
Los niños y niñas disponen de diversos vasos opacos vacíos y distintos \\
materiales: colonia, vinagre, hierbabuena, naranja, café, etc. Preparan \\
vasos con estos materiales para después mezclarlos y adivinar qué hay \\
en cada vaso, reconociendo las imágenes de las tarjetas.
\end{tabular}

¿Cómo podemos hacer nuestra colonia?

Se ofrece a los niños y niñas diversos botes, alcohol y elementos naturales, además de varios morteros. A través de la experimentación crean sus propias colonias.

¿Quiénes son pareja?

Materiales

1.Bolsas de tela; 2. Lana; 3.

Plantas aromáticas frescas:

Lavanda, romero,

hierbabuena, etc.

1.Vasos opacos; 2. Materiales

diversos: vinagre, café,

naranja, colonia, etc.; 3 .

Tarjetas con imágenes de los diferentes materiales.

1.Alcohol; 2. Botes vacíos; 3.

Elementos naturales: flores,

romero, canela, tomillo, albahaca, etc.

En ocho vasos opacos se introducen algodones impregnados en distintos olores: colonia, vinagre, limón y naranja, de modo que haya cuatro olores diferentes y se repita un mismo olor dos veces. Así habría dos vasos con algodón impregnado en colonia, otros dos en vinagre, otros dos en limón y dos en naranja. Los niños y niñas al oler el contenido de cada vaso deben ir localizando las parejas que huelen igual.

1.Vasos opacos; 2. Algodón impregnado de: limón, vinagre, colonia, naranja y limón.

Fuente: Elaboración propia

\subsection{Implementación y metodología de análisis}

Para cada uno de los cinco rincones que integran esta propuesta didáctica se siguió la misma dinámica:

- Asamblea de presentación y detección de ideas iniciales. En ella se realizaban diferentes cuestiones para conocer las concepciones del alumnado y, a continuación, se procedía a presentar el rincón correspondiente. Preguntas tales como ¿Con qué vemos? ¿Cómo reconocemos los diferentes colores? ¿Cómo reconocemos los diversos olores? ¿Cómo averiguamos a qué sabe algo? ¿Cómo sabemos que algo es rugoso? fueron algunas de las formuladas durante las asambleas de presentación.

- Trabajo libre por parte del alumnado en el rincón. Se programaron diferentes actividades para cada uno de los rincones, de forma que cada día se planteaba al alumnado la cuestión o cuestiones a indagar y se dejaba que exploraran y experimentaran autónomamente para darle respuesta.

- Puesta en común del trabajo realizado diariamente en el rincón. Al finalizar la jornada, se realizaba una puesta en común en la que los niños compartían los resultados que habían obtenido tras el trabajo libre.

- Asamblea final o de cierre. Una vez realizadas todas las actividades previstas en el rincón, se volvía a plantear al alumnado las preguntas de la asamblea inicial y se comentaban las conclusiones obtenidas tras el trabajo realizado.

Para registrar los posibles avances de aprendizaje se llevó, por un lado, un diario de clase, que permitió registrar, además de cuestiones actitudinales y procedimentales ante el desarrollo de las actividades, también, cuestiones cognitivas como las preguntas e hipótesis, que formularon los propios niños durante las mismas. Y, también, se cumplimentó una escala de observación al final de cada actividad. Esta escala de doble entrada nos permitió cuantificar las respuestas bajo criterios 
valorativos en base a su ejecución atendiendo a categorías según finalidad de la actividad (en Anexo mostramos la escala valorativa que se usó para el registro).

\section{RESULTADOS}

Los diferentes rincones diseñados han resultado atractivos para el alumnado, llamando su atención por la multitud de materiales disponibles y por las cuestiones planteadas. Las asambleas iniciales resultaron claves para ajustar las actividades previstas a las ideas expresadas. Al inicio de la propuesta los niños se mostraban algo perdidos y demandaban con frecuencia la presencia de la docente para verificar que lo que hacían era correcto. Esto fue cambiando conforme entraron en la dinámica propia de las actividades libres, de forma que ya en el segundo rincón prácticamente no demandaron atención de la docente $\mathrm{y}$, a partir del tercero, experimentaron con total libertad sin ninguna dificultad. Observamos también, de forma general, una tendencia a la exploración individual que se tornaba en grupal conforme avanzaba la propuesta didáctica.

Para cada uno de los rincones podemos destacar los siguientes resultados:

\section{Rincón del tacto}

Al inicio el alumnado presentó cierta dificultad para asociar el sentido del tacto a la piel, pero con las actividades planteadas a través de la experimentación se superó este obstáculo. Al ser el primer rincón que se realizó, algunas de las actividades que tenían un matiz más grupal resultaron algo más complejas. Este es el caso de la actividad de clasificación de los materiales, en la que debían ponerse de acuerdo para establecer los criterios que les permitieran clasificar los elementos. Tales como dureza, percepción agradable/desagradable (suave y áspero). Así la docente colaboró ayudándolos a tomar las decisiones. Para ello se intervino planteando cuestiones y ayudando a determinar el procedimiento a seguir en base a lo que cada niño iba expresando. Como se muestra en el Gráfico 1, la mayoría del alumnado lo desarrolla satisfactoriamente.

GRÁFICO 1. Distribución del alumnado según indicadores de logro en el rincón del tacto

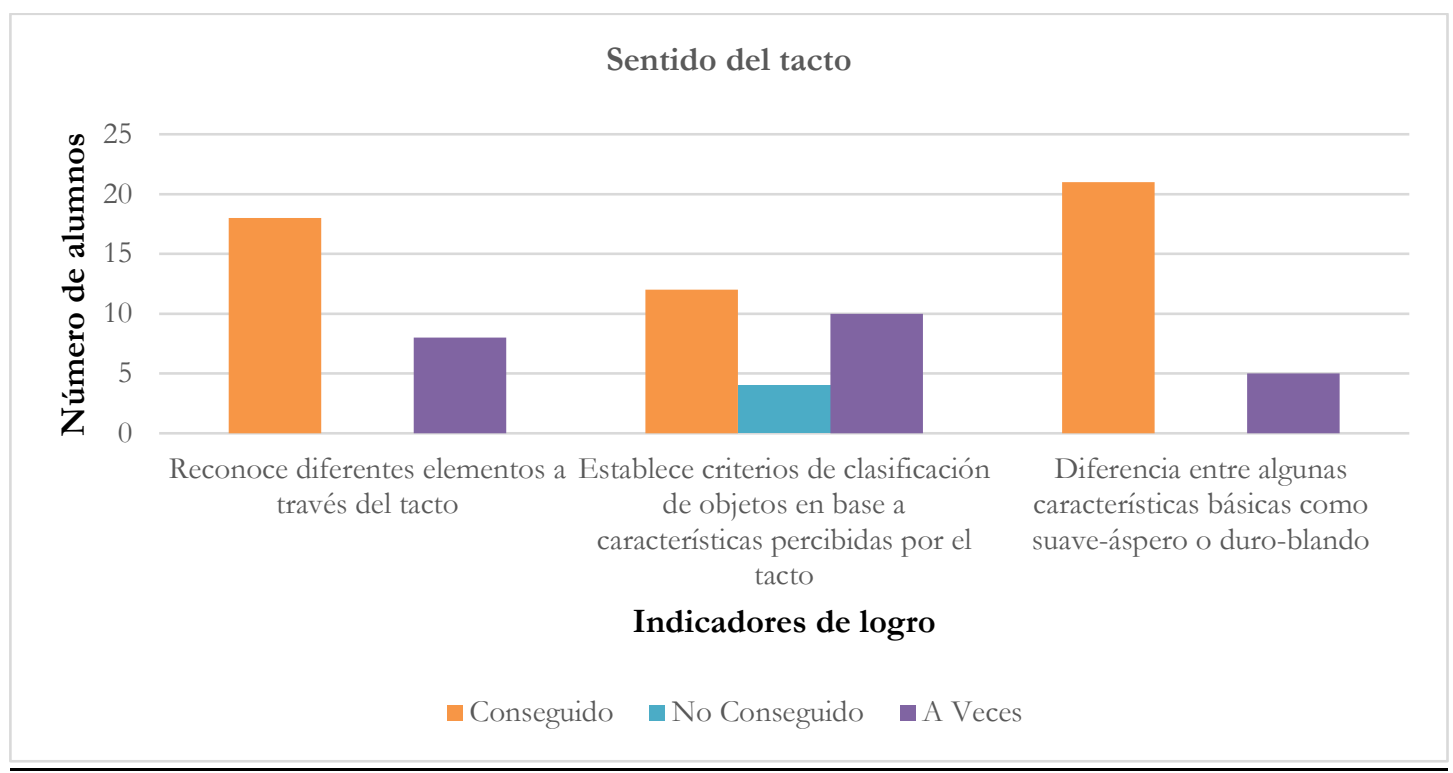

Fuente: Elaboración propia

Resulta interesante destacar el tiempo que dedicaban muchos de los alumnos a la manipulación de los materiales, así en la actividad del barro, pasaron bastante tiempo manipulando el aceite, tocando el barro o la vaselina. Las mezclas entre algunos de estos elementos también fueron de gran 
interés, incluso algunos olvidaron el objetivo de la actividad y se dedicaron a realizar mezclas entre elementos. Esto se aprovechó para introducir nuevas cuestiones de investigación relacionadas con los tipos de mezcla y las propiedades de ciertos materiales.

\section{Rincón de la vista}

En este rincón el alumnado mostró especial entusiasmo en la actividad de lupa y en la de la mesa de luz, por tratarse de recursos que no habían utilizado con anterioridad. Respecto a la lupa y, por su novedad, se hizo una breve asamblea antes de la actividad para tratar de conocer para qué se usa una lupa, estableciendo diferencias con otros instrumentos como microscopios o incluso telescopios. Aunque se ofreció una bandeja con materiales diversos, los propios niños localizaban nuevos objetos que observar e incluso en diversas ocasiones se miraban entre ellos para ver cómo se veían a través de la lupa diferentes partes de sus cuerpos. A partir de la experiencia, destacamos como en más de una ocasión, el alumnado llevaba al aula insectos del recreo para verlos a través de la lupa (ver Figura 3).

La actividad de las botellas se resolvió con cierta rapidez y no provocó demasiadas interacciones entre el alumnado, ya que se solían organizar por parejas en torno a las tres botellas que se habían creado, trabajando cada pareja de forma independiente. Encontraron dificultad para localizar los objetos de menor tamaño como el botón y algunas parejas finalizaban sin encontrar este objeto.

Por su parte, la actividad de la mesa de luz hizo que surgieran diversas cuestiones relativas a los huesos del cuerpo, surgiendo así un nuevo centro de interés. Al principio utilizaron la mesa de luz para ver las radiografías, pero pronto comenzaron a probar con sus propias manos para ver lo que ocurría con la luz y llevaron a esta mesa diferentes objetos del aula y del recreo.

Finalmente, en la actividad de creación de colores el alumnado no siempre probaba en la rueda de colores y después experimentaba con la pintura de dedos. La mayoría se decantaba por uno de los dos procesos y ahí exploraba posibilidades. Puesto que los materiales del rincón estaban accesibles siempre, a un niño se le ocurrió utilizar la mesa de luz para ver mejor los colores de la rueda y así se incorporó esta herramienta a la actividad.

Fruto de estas experiencias resulta que, como muestra el Gráfico 2, la mayoría del alumnado consigue reconocer y combinar colores, además de una eficaz manipulación de la instrumentación. No obstante, muestra mayor dificultad para reconocer objetos observando solo una parte de ellos.

GRÁFICO 2. Distribución del alumnado según indicadores de logro en el rincón de la vista

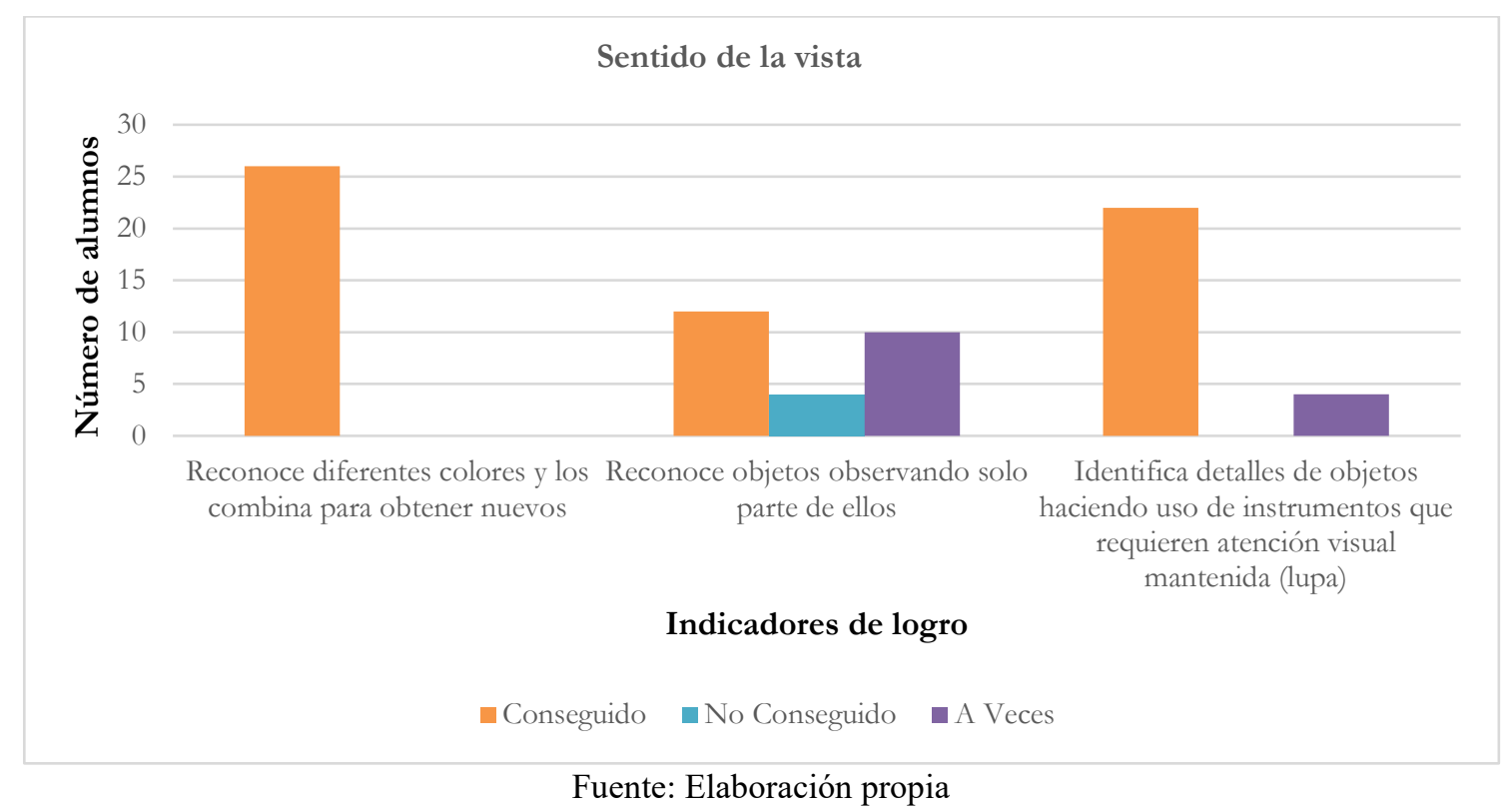




\section{Rincón del oído}

En este rincón los alumnos han ido discriminando diferentes tipos de sonido, tomando conciencia de que no todos los objetos pueden provocar sonidos. Como consecuencia de la actividad en la que cada alumno experimentó sobre la búsqueda de sonidos con diferentes objetos, se trabajó la clasificación de sonidos agradables y desagradables. La actividad del fonendoscopio les resultó altamente interesante y favoreció la interacción entre ellos. Los alumnos jugaron a escuchar sus propios latidos, su respiración e incluso lo utilizaron como un micrófono para escucharse a sí mismos. En otras ocasiones escuchaban los latidos de algún compañero simulando que era la consulta de un médico o jugaban con él como si fuera un teléfono donde uno escuchaba y otro hablaba por la campana del fonendoscopio.

En el juego de los botes encontraron cierta dificultad para recordar los objetos que estaban dentro y, por lo tanto, poder identificarlos por el sonido que producían al agitarlos. Para salvar esta dificultad se dejaron los materiales u objetos que había dentro al alcance de los niños, lo que ayudó a averiguar qué había dentro de los botes. El alumnado utilizó en diversas ocasiones los botes como maracas, haciéndolos sonar y creando ritmos agradables con ellos.

Finalmente, la actividad en la que se ofrecían diferentes cilindros y el alumnado experimenta con ellos comprobando si el sonido se trasmite igual con ellos o sin ellos, desencadenó diversas preguntas relacionadas con la transmisión del sonido y la amplificación de este, quedando registradas las siguientes: ¿El sonido mueve los cilindros? ¿Por qué con unos se escucha más fuerte que con otros? ¿El material del que está hecho el cilindro hace que se escuche más fuerte? ¿Y el tamaño del cilindro, afecta? Estas preguntas sirvieron para introducir la acción de vibrar y el concepto de vibración. Además, favoreció la interacción entre el alumnado, que se ayudaba para explorar distintas posibilidades con los diversos materiales.

El trabajo en este rincón, llevó a que todo el alumnado fuera capaz de diferenciar entre sonidos considerados agradables y desagradables. Del mismo modo, una amplia mayoría era capaz de producir sonidos y ritmos diversos con distintos materiales y logró asociar los sonidos con los objetos que los producían (ver Gráfico 3).

GRÁFICO 3. Distribución del alumnado según indicadores de logro en el rincón del oído

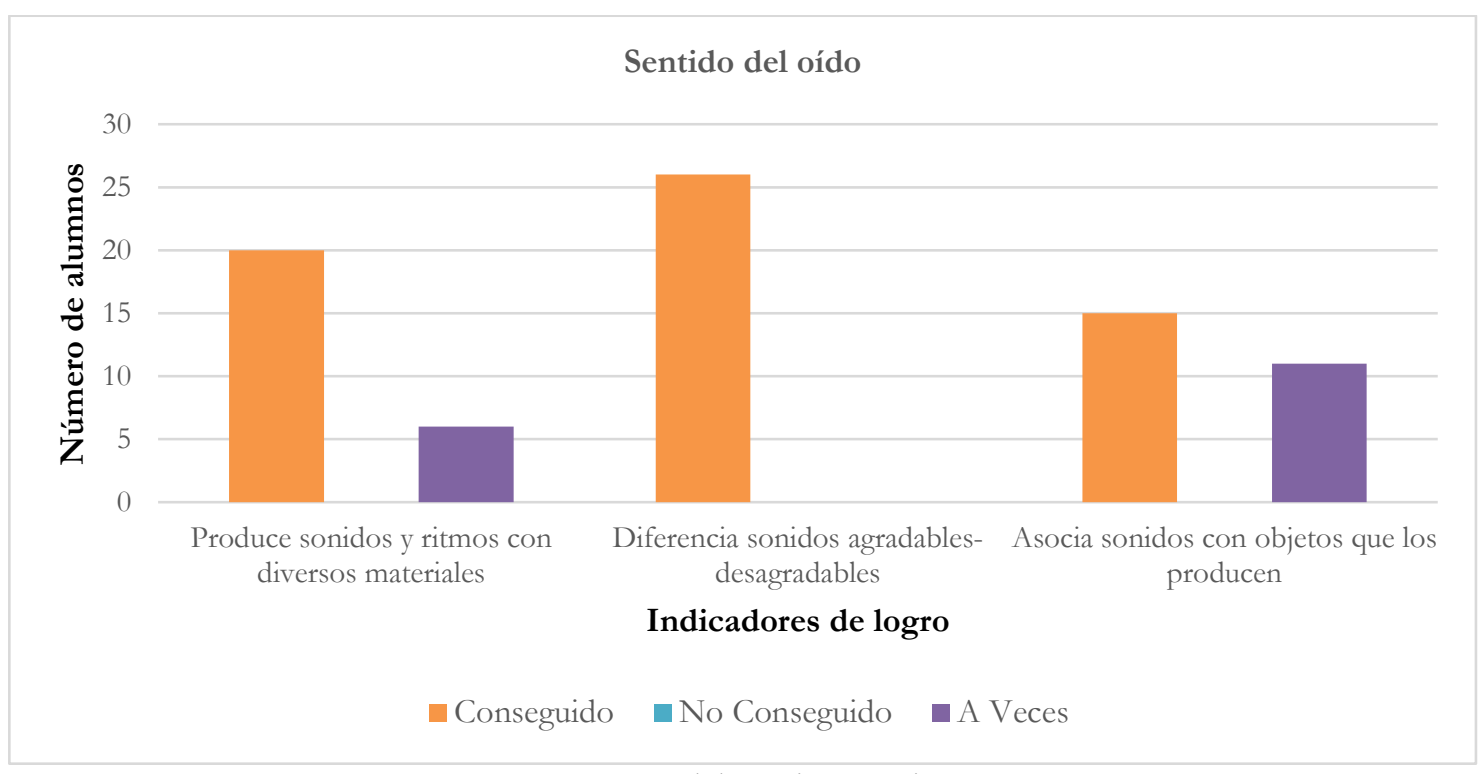

Fuente: Elaboración propia 


\section{Rincón del gusto}

En este rincón, al inicio, nos encontramos con algunos niños un tanto reacios a probar alimentos que no habían comido con anterioridad, obstáculo que se fue superando con la ayuda del resto, que animaban a sus compañeros. En la actividad en la que debían clasificar los alimentos, encontramos clasificaciones bastante dispares que no siempre respondían al sabor, por ejemplo, se hicieron clasificaciones en base al color o la forma, por lo que, en la asamblea tras la actividad, se aclararon ideas y se clarificaron conceptos. Sin embargo, hubo otras clasificaciones, que, sin responder a la esperada, tenía cierta lógica y se argumentó por parte del alumnado considerándose igualmente válidas, hablamos de clasificaciones que respondían a texturas o percepción en la boca.

En la actividad de los zumos, los alumnos encontraron cierta dificultad para hallar la correspondencia entre el vaso y la fruta, normalmente localizaban un par de parejas que estaban formada por las frutas más comunes. Discutían entre ellos y trataban de ponerse de acuerdo, recurriendo en más de una ocasión a levantar la tapa del vaso o a volcar el contenido de este para que esto les ayudara a identificar el contenido. Esto se aprovechó para trabajar la complementariedad de los sentidos.

La actividad donde se cambiaba el sabor del agua fue algo compleja y no todos los grupos entendieron desde el inicio el sentido. Sin embargo, una vez que lograban entender bien la pregunta planteada, comenzaban a probar y trataban de ir sacando conclusiones, tales como: "Si le echamos sal sabe como el agua del mar, pero si le echamos azúcar sabe diferente. El agua va cambiando su sabor porque sola no sabe a nada.”. La breve asamblea final resultó esencial para lograr conclusiones coherentes.

Por último, en la actividad de los yogures, se formularon al inicio algunas hipótesis que pudieron ir comprobando mediante la experimentación. De este modo, casi todos los niños creían al inicio que el colorante haría que el yogur cambiara de sabor, así asociaban, por ejemplo, el yogur de color rosa con la fresa o el de color amarillo con el limón. Sin embargo, las experimentaciones los llevaron a ver que esto no era cierto en su caso y que todos sabían igual. De nuevo, trabajamos en base a estas ideas las relaciones entre los sentidos. A pesar de esto, podemos ver en el Gráfico 4, que resulta complejo para el alumnado superar la asociación entre características visuales (color) de los elementos y los sabores. Sin embargo, las actividades realizadas hacen posible que la gran mayoría del alumnado diferencie sabores dulces y saldos, así como que sea capaz de reconocer alimentos a través del sabor.

GRÁFICO 4. Distribución del alumnado según indicadores de logro en el rincón del gusto

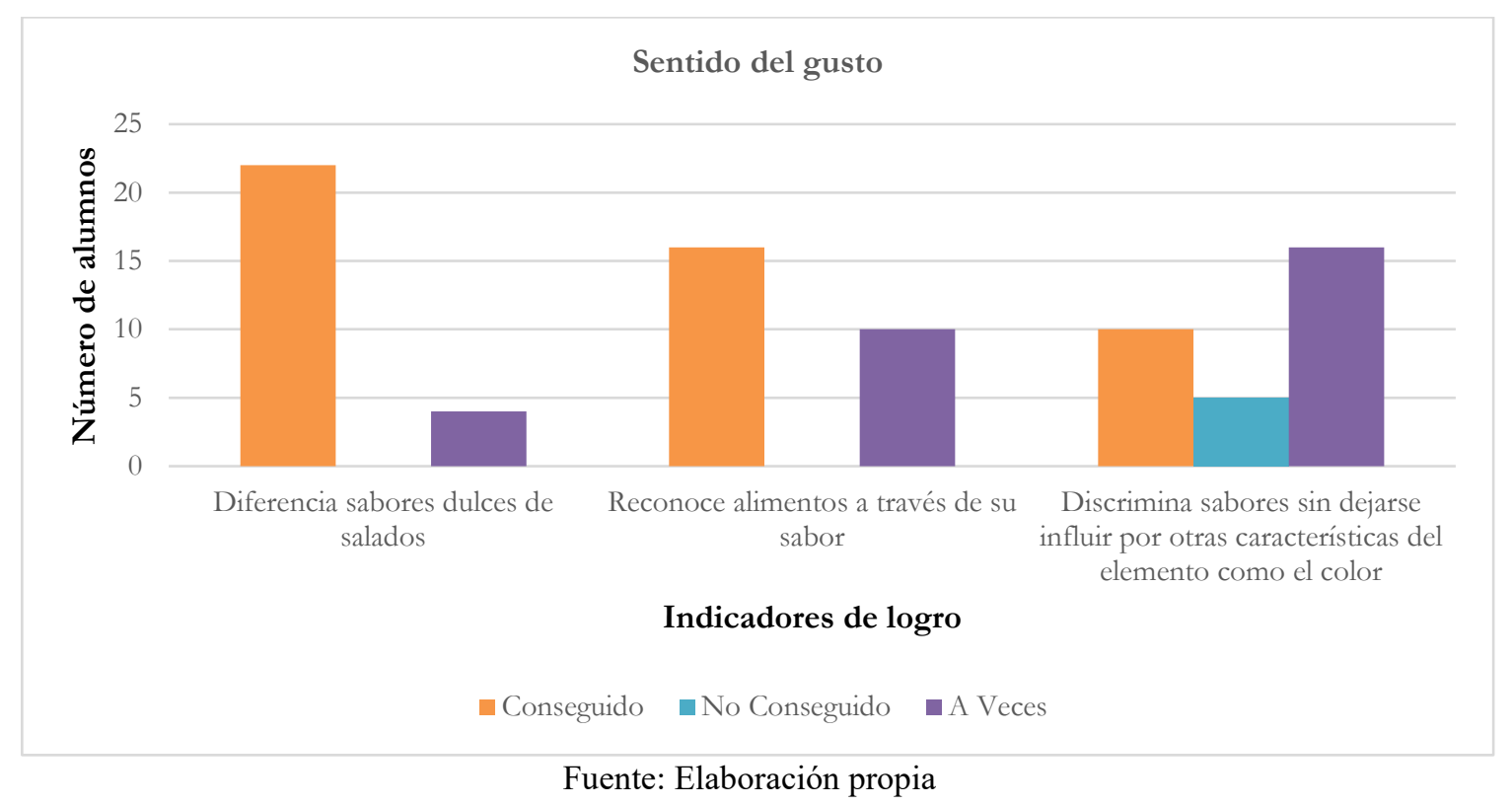




\section{Rincón del olfato}

La actividad de las bolsitas aromáticas se desarrolló con bastante agilidad, los niños eligieron aquellas plantas que más le gustaban y procedían a llenar con ellas sus bolsitas.

En la actividad de ¿A qué huele?, el alumnado experimentó con los distintos materiales, discriminando olores, comparando y eligiendo en función de los gustos. Creados los vasos, jugaron adivinando lo que había en su interior, recurriendo a veces al sonido para ayudarse, oportunidad que se utilizó para trabajar de nuevo la complementariedad de los sentidos.

La creación de colonias fue de gran interés, porque, aunque se desconocía el proceso exacto, establecieron hipótesis, tal como "Necesitamos agua muy muy caliente para que, al echar las flores, estas se deshagan y así ya sale el perfume", y fueron comprobando lo que ocurría. Así llegaron a crear colonias muy diferentes. En esta actividad, fruto de las mezclas que algunos alumnos realizaron, pudimos abordar la idea de olores agradables y desagradables, así como las posibilidades de preferencia por parte de cada individuo.

Para terminar, apuntamos que la actividad de emparejar vasos con algodón impregnando en diferentes sustancias fue algo compleja, confundiendo en numerosas ocasiones el limón y la naranja y recurriendo a abrir los vasos en busca de alguna pista que les ayudara a encontrar las parejas. Los alumnos se fueron ayudando entre ellos, escuchándose y comparando sus decisiones para lograr una respuesta conjunta a la actividad. Finalmente, podemos decir que el alumnado logró con facilidad diferenciar olores agradables y desagradables, encontrando algunas dificultades para identificar semejanzas y diferencias entre olores y siendo para ellos más complejo el seguir una secuencia de pasos para obtener olores diferentes (Gráfico 5).

GRÁFICO 5. Distribución del alumnado según indicadores de logro en el rincón del olfato

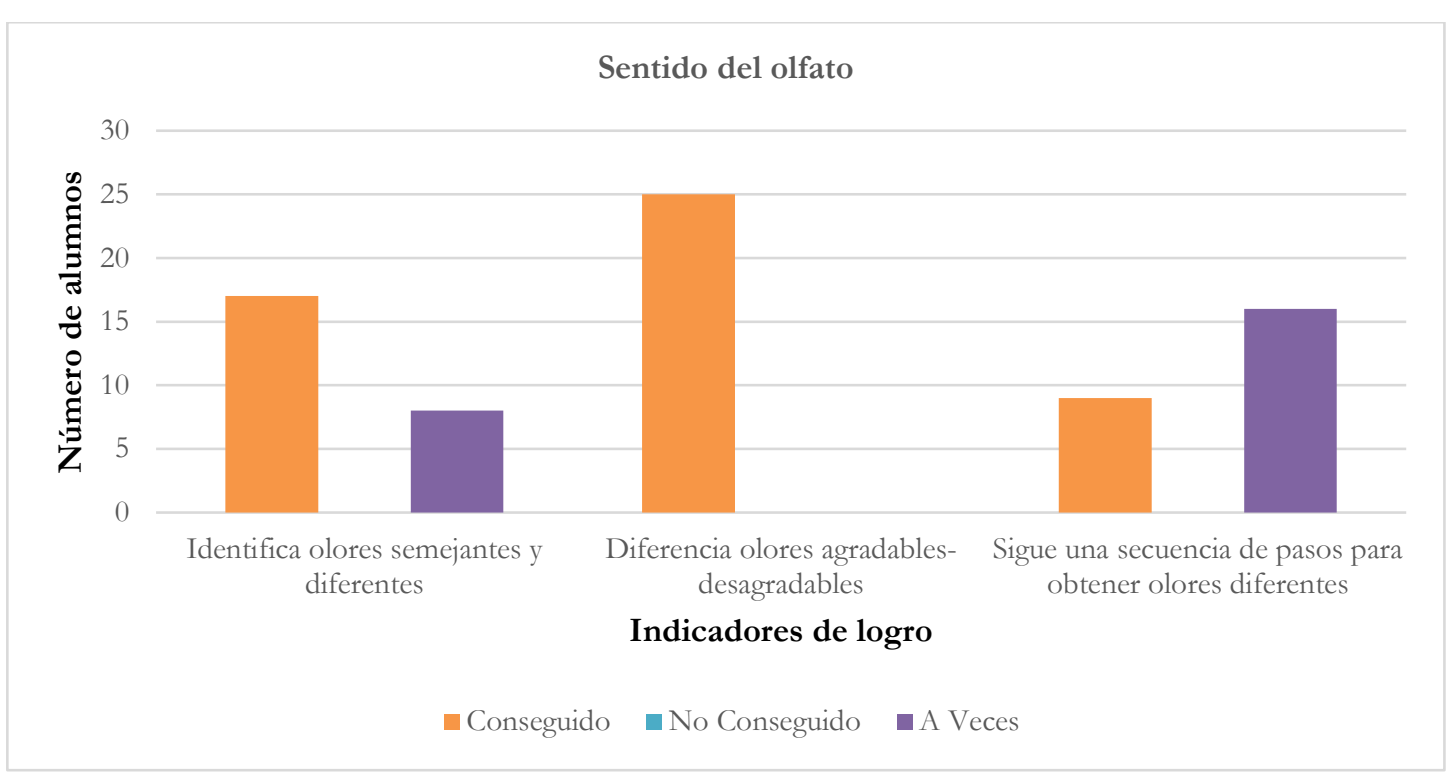

Fuente: Elaboración propia

\section{Conclusiones}

La propuesta didáctica realizada ha logrado cubrir los objetivos planteados y nos ha brindado la oportunidad de introducir a los más pequeños en la experimentación y la indagación de forma autónoma. Los sentidos es un tema que suele abordarse con frecuencia en Educación Infantil, pero consideramos que, generalmente, no se ofrece a los niños la posibilidad de experimentar a través de ellos y reflexionar sobre lo ocurrido. Sin embargo, esta propuesta, a pesar de que podría mejorarse, incorporando actividades aún más abiertas y revisando aquellos aspectos que han resultado de una complejidad excesiva para el alumnado, ha abordado este tema con mayor profundidad y durante su 
desarrollo hemos podido comprobar su repercusión en los conocimientos, las destrezas y las actitudes de nuestros escolares. La implicación y la motivación han ido incrementándose conforme avanzaba la propuesta. Coincidimos con Hidalgo-Navarrete, De De, Risueño, Montijano y Perales (2009) en la importancia de que el aprendizaje se construya en el seno de proyectos globalizadores que, como el nuestro, permiten abordar las tres áreas del currículum de Educación Infantil alrededor de una misma temática (los sentidos) y no mediante actividades aisladas para adquirir esquemas conceptuales científicos. Así, los niños se han mostrado cada vez más capaces de experimentar, de lanzar hipótesis, de comprobarlas y de reflexionar sobre ello, coincidente con resultados alcanzados por estudios como el de Hidalgo et al. (2009) o Paños, Ruiz-Gallardo y Mateos (2018). En este sentido, consideramos clave el carácter abierto de las actividades y las preguntas que han iniciado cada una de ellas, ya que abrían al alumnado múltiples posibilidades, mostrándoles todo un mundo por descubrir. Sobre esto, comprobamos que su desarrollo provoca en el alumnado cuestiones muy interesantes que pueden tomarse como preguntas de partida para la ampliación de estos rincones o para el diseño de un proyecto en sí, como por ejemplo sobre la ciencia de los materiales, su geometría y sus características a raíz de la experimentación del sonido o del tacto (deformación, elasticidad, viscosidad...). Esta propuesta nos brinda una futura línea de intervención que conecta y profundiza la temática abordada. La secuencia de actividades ha resultado, además, sencilla en su implementación y se ha llevado a cabo con materiales de fácil acceso y cotidianos para los escolares, aspectos esenciales en la experimentación en ciencias en esta etapa (Brown, 1991).

Creemos por ello, que esta propuesta ha logrado que los más pequeños conecten con la ciencia y de manera autónoma aprendan a través de ella a descubrir el mundo que les rodea, aportándoles, además, una visión de la ciencia capaz de generarles emociones.

\section{Referencias}

Andiema, N.C. (2016). Effect of Child Centred Methods on Teaching and Learning of Science Activities in Pre-Schools in Kenya. Journal of Education and Practice, 7(27), 1-9.

Ansari, A., y Purtell, K. (2017). Activity settings in full-day kindergarten classrooms and children'searly learning. Early Childhood Research Quarterly, 38, 23-32. DOI: 10.1016/j.ecresq.2016.09.003

Aranda, R. E. (2008). Atención temprana en educación infantil. Madrid: Wolters Kluwer.

Bergen, D. (2002). The role of pretend play in children's cognitive development. Early Childhood Research \& Practice, 4(1).

Bermejo, C. R., y Blázquez, C. T. (2016). El juego infantily su metodología. Madrid: Editorial Síntesis. Blanco-Carabias, E., López-Luengo, M.A., Vallés, C., y Gil, C. (2018). Ciencia e inclusión en Educación Infantil. Actas 28 Encuentros de Didáctica de las Ciencias Experimentales, 11151120.

Brown, S. E. (1991). Experimentos de Ciencias en educación infantil. Narcea Ediciones.

Cabello, M. J. (2011). Ciencia en educación infantil: La importancia de un "rincón de observación y experimentación" o "de los experimentos" en nuestras aulas. Pedagogía Magna, 10, 58-63.

Córdoba, D. (2011). Desarrollo cognitivo, sensorial, motor y psicomotor en la infancia. Antequera, Málaga: Innovación y Cualificación.

Cruz-Guzmán, M. (2018). ¡Vivimos en la Tierra y exploramos el Universo! En M. Puig y F. RodríguezMarín (Coord.) La enseñanza del Entorno en Educación Infantil. Proyectos y rincones (pp. 145-163). Madrid: Pirámide.

Cruz-Guzmán, M., García-Carmona, A., y Criado, A. M. (2017). Aprendiendo sobre los cambios de estado en educación infantil mediante secuencias de pregunta-predicción- comprobación experimental. Enseñanza de las Ciencias, 35(3), 175-193. DOI: 10.5565/rev/ensciencias. 2336 
De la Blanca, S., Hidalgo, J., Burgos, C. (2013). Escuela infantil y ciencia: la indagación científica para entender la realidad circundante. Enseñanza de las Ciencias, $\mathrm{n}^{\mathbf{0}}$ extraordinario, 979-983.

Diachenko, O. M. (2011). “On Major Developments in Preschoolers' Imagination.” International Journal of Early Years Education, 19(1), 19-25. DOI:10.1080/09669760.2011.570996.

Epstein, A. S. (2014). The Intentional Teacher: Choosing the Best Strategies for Young Childrens Learning (Rev. Ed). Washington D.C.: NAEYC.

Eshach, H., y Fried, M. N. (2005). Should science be taught in early childhood? Journal of Science Education and Technology, 14(3), 315-336. DOI: 10.1007/s10956-005-7198-9.

Fernández-Arroyo, J., López-Lozano, L., y García-Díaz E. (2018). Iniciando la construcción de conciencia ecológica desde la Educación Infantil. El uso de los huertos escolares. En M. Puig y F. Rodríguez-Marín (Coord.) La enseñanza del Entorno en Educación Infantil. Proyectos y rincones (pp. 187-203). Madrid: Pirámide.

Fernández-Manzanal, R., y Rodríguez-Barreiro, L. M. (2006). Los pequeños de cuatro años en el rincón de ciencias: qué ven y qué dicen sobre el nacimiento de las plantas. Alambique: Didáctica de las Ciencias Experimentales, 49, 105-113.

Fernández-Oliveras, A., Molina, V., y Oliveras, M. L. (2016). Estudio de una propuesta lúdica para la educación científica y matemática globalizada en infantil. Revista Eureka sobre Enseñanza y Divulgación de las Ciencias, 13 (2), 373-383.

Hidalgo-Navarrete, J., De De, S., Risueño, J., Montijano, B., y Perales, R. (2009). Desarrollo de competencias básicas desde las ciencias en la educación infantil y la educación de adultos. Enseñanza de las ciencias, $\mathrm{N}^{\circ}$ Extra, 2163-2166.

García-Carmona, A., Criado, A. M., y Cañal, P. (2014). Alfabetización científica en la etapa 3-6 años: un análisis de la regulación estatal de enseñanzas mínimas. Enseñanza de las Ciencias, 32(2), 131-149.

Gómez-Crespo, M. A. (2003. El rincón de los experimentos. Aula de innovación educativa, 126, 2830 .

Gómez-Montilla, C., y Ruiz-Gallardo, J. R. (2016). El rincón de la ciencia y actitud hacia las ciencias en educación infantil. Revista Eureka sobre Enseñanza y Divulgación de las Ciencias, 13 (3), 643-666.

Johansson, E., y Løkken G. (2014). Sensory Pedagogy: Understanding and encountering children through the senses. Educational Philosophy and Theory, 46. Recuperado de http://www.tandfonline.com/doi/full/10.1080/00131857.2013.783776

Laguía, M. J., y Vidal, C. (2011). Rincones de actividad en la escuela infantil (0-6 años). Barcelona: Graó.

Madrid, D., y Mayorga, M. J. (2012). La organización del espacio en el aula de infantil. Barcelona: Octaedro.

Mateo, E., Ferrer, L. M., Mazas, B., Hervás, A., y Muñoz A. (2017). ¿Qué hay dentro de la cueva?: una experiencia multisensorial para trabajar las ciencias con alumnos de educación infantil. Enseñanza de las Ciencias, $\mathrm{n}^{\mathrm{o}}$ extraordinario, 951-957.

Martín, J. (2008). Organización y funcionamiento de rincones en educación infantil. Revista digital Innovación $y$ Experiencias Educativas, 13. Recuperado de http://www.csicsif.es/andalucia/modules/modense/revista/pdf/Numero13/JOSEFA_MARTIN_ 1.pdf.

Medrano, G., Bello, L., y Fernández, R. (2006). Las actividades en el rincón de ciencias. Un pretexto para la globalización en la etapa infantil. Aula de infantil, 29, 28-30.

Moreno, F. M. (2015). La utilización de los materiales como estrategia de aprendizaje sensorial en infantil. Opción, 31(2), 772-789.

Paños, E., Ruiz-Gallardo, J.R., y Mateos, A. (2018). Examinando legumbres. Una propuesta experimental en Educación Infantil. Actas 28 Encuentros de Didáctica de las Ciencias Experimentales, 269-274. 
Ramos, M. M., y Pérez, C. (2009). El rincón de los gusanos de seda. Cuadernos de Pedagogía, 387, 28-30.

Rodríguez-Marín, F. y Puig, M. (2018). El área de conocimiento del entorno a través de proyectos y rincones. En M. Puig y F. Rodríguez-Marín (Coord.) La enseñanza del Entorno en Educación Infantil. Proyectos y rincones (15-26). Madrid: Pirámide.

Rogers, S. (Ed.). (2011). Rethinking play and pedagogy in early childhood education: Concepts, contexts and cultures. London, England, New York, NY: Routledge.

Saçkes, M., Trundle, K. C., Bell, R. L., y O’Connell, A. A. (2011). The influence of early science experience in kindergarten on children's immediate and later science achievement: Evidence from the early childhood longitudinal study. Journal of Research in Science Teaching, 48(2), 217-235. DOI: 10.1002/tea.20395

Singer, D., Golinkoff, R., y Hirsh-Pasek, K. (Ed.). (2006). Play = learning: How play motivates and enhances children's cognitive and social-emotional growth. New York, NY: Oxford University Press.

Torres-Porras, J., Alcántara, J., Arrebola, J.C., Rubio, S.J., y Mora, M. (2017). Trabajando el acercamiento a la naturaleza de los niños y niñas en el Grado de Educación Infantil. Crucial en la sociedad actual. Revista Eureka sobre Enseñanza y Divulgación de las Ciencias, 14(1), 258270.

Zacharias, C. Loizou, E., y Papaevripidou, M. (2012). Is physicality an important aspect of learning through science experimentation among kindergarten students? Early Childhood Research Quarterly, 27, 447-457. DOI: 10.1016/j.ecresq.2012.02.004

\section{Cómo CITAR ESTE ARTÍ́CULO}

Puig Gutiérrez, M., López-Lozano, L. y García Rodríguez, R. (2020). Experimentando con los sentidos: un rincón de ciencias en Educación Infantil. Didáctica de las ciencias experimentales y sociales, 39, 117-134. DOI: 10.7203/DCES.39.16893. 


\section{ANEXO}

TABLA 6. Escala valorativa rincón del tacto

\begin{tabular}{|c|c|c|c|c|}
\hline \multirow{2}{*}{ Actividades } & \multirow{2}{*}{ Indicadores de logro } & \multicolumn{3}{|c|}{ Niveles de consecución } \\
\hline & & Conseguido & A veces & No conseguido \\
\hline $\begin{array}{l}\text { ¿Cómo puedo } \\
\text { volver blando un } \\
\text { trozo de barro } \\
\text { duro? }\end{array}$ & $\begin{array}{l}\text { Diferencia entre algunas } \\
\text { características básicas como } \\
\text { suave-áspero o duro/blando }\end{array}$ & & & \\
\hline $\begin{array}{l}\text { ¿Qué hay en la } \\
\text { caja de las } \\
\text { sorpresas? }\end{array}$ & $\begin{array}{l}\text { Reconoce diferentes elementos a } \\
\text { través del tacto }\end{array}$ & & & \\
\hline \multirow[t]{2}{*}{$\begin{array}{l}\text { ¿Qué hay dentro } \\
\text { de los globos? }\end{array}$} & $\begin{array}{l}\text { Reconoce diferentes elementos a } \\
\text { través del tacto }\end{array}$ & & & \\
\hline & $\begin{array}{l}\text { Diferencia entre algunas } \\
\text { características básicas como } \\
\text { suave-áspero o duro/blando }\end{array}$ & & & \\
\hline $\begin{array}{l}\text { ¿Cómo podemos } \\
\text { clasificar } \text { estos } \\
\text { materiales? }\end{array}$ & $\begin{array}{l}\text { Establece criterios de } \\
\text { clasificación de objetos en base a } \\
\text { características percibidas por el } \\
\text { tacto }\end{array}$ & & & \\
\hline
\end{tabular}

TABLA 7. Escala valorativa rincón de la vista

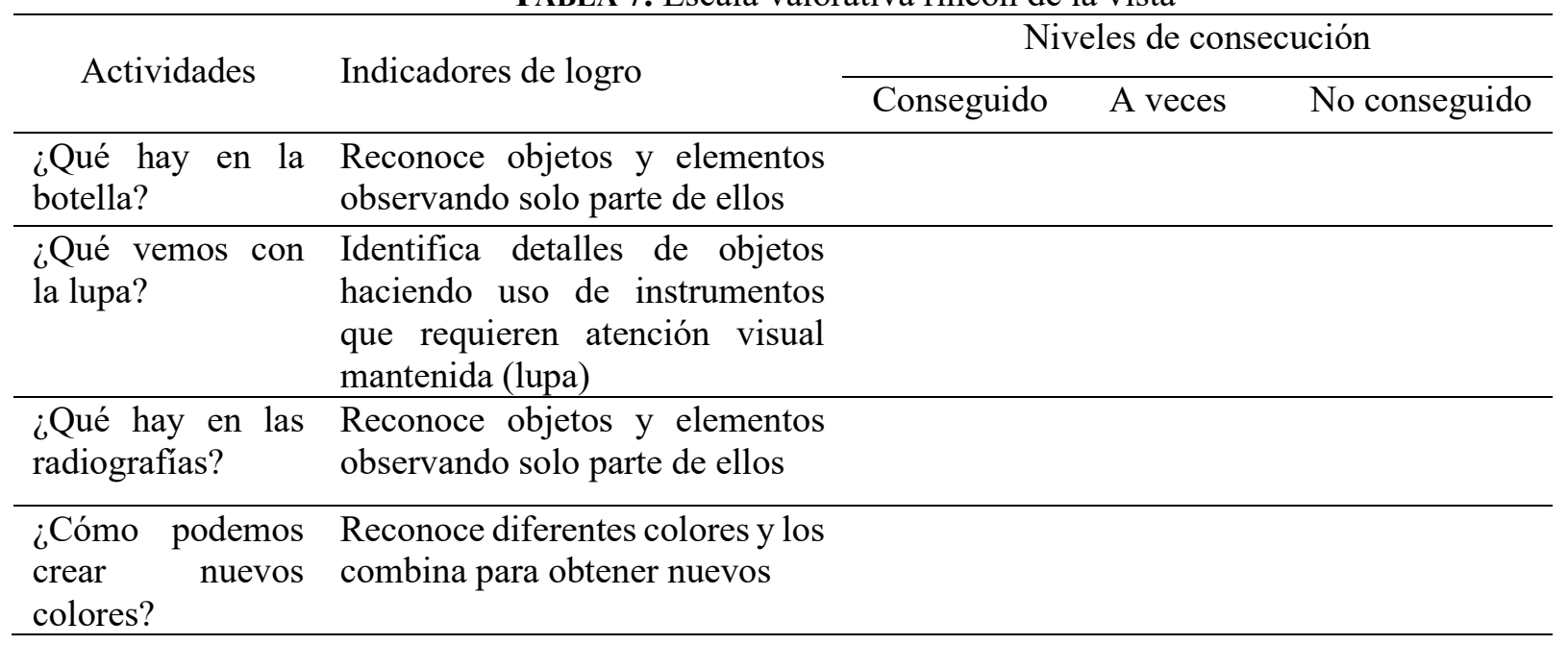


TABLA 8. Escala valorativa rincón del oído

\begin{tabular}{llll}
\hline \multirow{2}{*}{ Actividades } & Indicadores de logro & \multicolumn{2}{c}{ Niveles de consecución } \\
\cline { 3 - 4 } $\begin{array}{l}\text { ¿Todos los objetos } \\
\text { producen } \\
\text { sonidos? }\end{array}$ & $\begin{array}{l}\text { Produce e identifica sonidos y } \\
\text { ritmos con diversos materiales y } \\
\text { elementos }\end{array}$ & \\
\hline ¿Qué sonidos & $\begin{array}{l}\text { Produce e identifica sonidos y } \\
\text { produce } \\
\text { cuerpo? }\end{array}$ & $\begin{array}{l}\text { ritmos con diversos materiales y } \\
\text { elementos }\end{array}$ \\
\hline ¿Qué hay dentro? & $\begin{array}{l}\text { Asocia sonidos con objetos que } \\
\text { los producen }\end{array}$ \\
\hline ¿Cómo suenan? & $\begin{array}{l}\text { Produce e identifica sonidos y } \\
\text { ritmos con diversos materiales y } \\
\text { elementos }\end{array}$ \\
\cline { 2 - 3 } & $\begin{array}{l}\text { Diferencia sonidos agradables- } \\
\text { desagradables }\end{array}$ \\
\hline
\end{tabular}

TABLA 9. Escala valorativa rincón del gusto

\begin{tabular}{|c|c|c|c|c|}
\hline \multirow{2}{*}{ Actividades } & \multirow{2}{*}{ Indicadores de logro } & \multicolumn{3}{|c|}{ Niveles de consecución } \\
\hline & & Conseguido & A veces & No conseguido \\
\hline ¿A qué sabe? & $\begin{array}{l}\text { Diferencia sabores dulces de } \\
\text { salados }\end{array}$ & & & \\
\hline $\begin{array}{l}\text { ¿De qué es este } \\
\text { zumo? }\end{array}$ & $\begin{array}{l}\text { Reconoce alimentos a través de } \\
\text { su sabor }\end{array}$ & & & \\
\hline $\begin{array}{l}\text { ¿Cómo podemos } \\
\text { cambiar el sabor } \\
\text { del agua? }\end{array}$ & $\begin{array}{l}\text { Diferencia sabores dulces de } \\
\text { salados }\end{array}$ & & & \\
\hline $\begin{array}{lr}\text { ¿Cómo podemos } \\
\text { hacer que algo } \\
\text { cambie } \\
\text { aspecto, pero no } \\
\text { su sabor? }\end{array}$ & $\begin{array}{l}\text { Discrimina sabores sin dejarse } \\
\text { influir por otras características } \\
\text { del elemento como el color }\end{array}$ & & & \\
\hline
\end{tabular}

TABLA 10. Escala valorativa rincón del olfato

\begin{tabular}{|c|c|c|c|c|}
\hline \multirow{2}{*}{ Actividades } & \multirow{2}{*}{ Indicadores de logro } & \multicolumn{3}{|c|}{ Niveles de consecución } \\
\hline & & Conseguido & A veces & No conseguido \\
\hline $\begin{array}{l}\text { ¿Cómo podemos } \\
\text { hacer bolsitas de } \\
\text { olores diferentes? }\end{array}$ & $\begin{array}{l}\text { Identifica olores semejantes y } \\
\text { diferentes }\end{array}$ & & & \\
\hline ¿A qué huele? & $\begin{array}{l}\text { Diferencia olores agradables- } \\
\text { desagradables }\end{array}$ & & & \\
\hline $\begin{array}{lr}\text { ¿Cómo } & \text { podemos } \\
\text { hacer } & \text { nuestra } \\
\text { colonia? } & \end{array}$ & $\begin{array}{l}\text { Sigue una secuencia de pasos } \\
\text { para obtener olores diferentes }\end{array}$ & & & \\
\hline $\begin{array}{l}\text { ¿Quiénes son } \\
\text { pareja? }\end{array}$ & $\begin{array}{l}\text { Identifica olores semejantes y } \\
\text { diferentes }\end{array}$ & & & \\
\hline
\end{tabular}


